\title{
SPECKLE NOISE REDUCTION IN SAR IMAGES USING INFORMATION THEORY
}

\author{
D. Chan ${ }^{1}$, J. Gambini ${ }^{2 *}$ A.C.Frery ${ }^{3}$ \\ ${ }^{1}$ Universidad Tecnológica Nacional, Facultad Regional Buenos Aires, Ciudad Autónoma de Buenos Aires, Argentina. email: \\ mchan@frba.utn.edu.ar \\ ${ }^{2}$ Departamento de Ingeniería Informática, Instituto Tecnológico de Buenos Aires, Buenos Aires, Argentina and Depto. de Ingeniería en \\ Computación, Universidad Nacional de Tres de Febrero, Pcia. de Buenos Aires, Argentina. email: mgambini@itba.edu .ar \\ ${ }^{3}$ Laboratório de Computação Científica e Análise Numérica, Universidade Federal de Alagoas, Brazil. email: acfrery@laccan.ufal . br
}

KEY WORDS: Speckle filter, $h-\phi$ Entropies, asymptotic variance

\begin{abstract}
:
In this work, a new nonlocal means filter for single-look speckled data using the Shannon and Rényi entropies is proposed. The measure of similarity between a central window and patches of the image is based on a statistical test for comparing if two samples have the same entropy and hence have the same distribution.

The results are encouraging, as the filtered image has better signal-to-noise ratio, it preserves the mean, and the edges are not severely blurred.
\end{abstract}

\section{INTRODUCTION}

The interference of waves reflected during the acquisition process of SAR (Synthetic Aperture Radar) images give rise to a multiplicative and non-Gaussian noise that characterizes them and is known as speckle noise (Oliver, Quegan, 2004). Many image filters are based on the parameter estimation of the underlying distribution of the data, but SAR image data have a heavy tailed and outliers prone distribution (Rojo, 2013, Gambini et al., 2015). Classic filters are not adequate to SAR images denoising, due to this noise.

Maximum a Posteriori Filters have been used to reduce the noise of single look SAR images in (Kuan et al., 1987), modeling the a priori distribution of the backscatter with a Gaussian law. In (Lopes et al., 1990, Sant'Anna, 1995) other a priori distributions, such as the $\beta$ and $\Gamma$, were utilized.

Stack filters have been studied that have shown that they preserve the edges and details (Dellamonica et al., 2007). Adaptive Stack filters have been proposed in (Lin et al., 1990) that were improved with fast algorithms in (Lin, Kim, 1994, Buemi et al., 2014).

In (Ferraioli et al., 2019) a nonlocal anisotropic despeckling approach for SAR images, is presented. This diversity of proposals generates the needing of to define criteria in order to evaluate the quality of a filter in a speckled image. In (Wang et al., 2002) the difficulty of measuring the quality of a filter, is analyzed. In (Gómez Déniz et al., 2017) as assessment of quality filter measures, is showed.

Nonlocal Means algorithms are a successful approach for image denoising. These filters take of a group of pixels surrounding a target pixel to smooth the image. In (Lebrun et al., 2013) a Bayes theorem based method, is presented.

The non-local means filtering takes a mean of a group of pixels in the image, weighted by values. Different methods apply a

\footnotetext{
${ }^{*}$ Corresponding author
}

diversity of criteria for defining the weight values. In (Torres et al., 2014) a speckle reduction method was developed for polarimetric SAR imagery by means of stochastic distances and nonlocal means.

Due to the stochastic nature of speckle noise, the statistical modeling of SAR data is strategic for image interpretation (Chan et al., 2019). In (Gao, 2010), the author details different models proposed for describing these kind of data and analyzes their properties. The multiplicative model for SAR image modeling appears to be an excellent choice. It states that the observed data can be modeled by a random variable $Z$, which is the product of two independent random variables: $X$ which describes the backscatter, and $Y$ that models the speckle noise. Following the multiplicative model, Frery et al. (Frery et al., 1997) introduced the $\mathcal{G}_{I}^{0}$ distribution which has been widely used for SAR data analysis. It is referred to as a Universal Model because of its flexibility and tractability (Mejail et al., 1998). It provides a suitable way for modeling areas with different degrees of texture, reflectivity and signal-to-noise ratio.

The $\mathcal{G}_{I}^{0}$ distribution is indexed by three parameters:

- $\alpha$, related to the target texture,

- $\gamma$, related to the brightness and called scale parameter,

- $L$, the number of looks, which describes the signal-tonoise ratio.

The two first may vary among positions, while the latter can be considered the same on the whole image. The third can be known or estimated.

Shannon (Shannon, 1948) proposed the entropy to measure the relative information between two random variables using their density functions. Entropies were studied by Kullback and Leibler (Kullback, Leibler, 1951) and Rényi (Rényi, 1961), among others. These measures are basilar elements for defining divergences. Liese and Vajda (Liese, Vajda, 2006) discussed the properties of several divergences. 
The Shannon entropy has been widely applied in statistics, image processing, and even in SAR image analysis (Nascimento et al., 2018).

In this work we use Shannon and Rényi entropies of the $\mathcal{G}_{I}^{0}$ distribution to develop our aim and we propose a nonlocal filter for speckled images noise reduction. We construct a filter of type non local means and employ explicit expressions of these entropies for the selection of the weights.

This article unfolds as follows. In Section 2, the $\mathcal{G}_{I}^{0}$ distribution is explained. Section 3 introduces the formulae of entropies for $\mathcal{G}_{I}^{0}$ distribution. In Section 4, the proposal algorithm for speckle reduction is detailed. In Section 5, the results of applying the method to synthetic images are presented. Finally, in Section 6 conclusions and future work are exhibited.

\section{THE $\mathcal{G}_{I}^{0}$ DISTRIBUTION}

Speckle noise follows a $\Gamma$ distribution, with density

$$
f_{Y}(y ; L)=\frac{L^{L}}{\Gamma(L)} y^{L-1} \exp \{-L y\},
$$

denoted by $Y \sim \Gamma(L, L)$. The physics of image formation imposes $L \geq 1$.

The model for the backscatter $X$, may be any distribution with positive support. Frery et al. (Frery et al., 1997) proposed using the Reciprocal Gamma law, a particular case of the Generalized Inverse Gaussian distribution, which is characterized by the density

$$
f_{X}(x ; \alpha, \gamma)=\frac{\gamma^{-\alpha}}{\Gamma(-\alpha)} x^{\alpha-1} \exp \{-\gamma / x\},
$$

where $\alpha<0$ and $\gamma>0$ are the texture and the scale parameters, respectively. Under the multiplicative model, the return $Z=$ $X . Y$ follows a $\mathcal{G}_{I}^{0}(\alpha, \gamma, L)$ distribution, whose density is

$$
f_{Z}(z)=\frac{L^{L} \Gamma(L-\alpha)}{\gamma^{\alpha} \Gamma(-\alpha) \Gamma(L)} \cdot \frac{z^{L-1}}{(\gamma+z L)^{L-\alpha}},
$$

where $-\alpha, \gamma, z>0$ and $L \geq 1$. The $r$-order moments of the $\mathcal{G}_{I}^{0}(\alpha, \gamma, L)$ distribution are:

$$
E\left(Z^{r}\right)=\left(\frac{\gamma}{L}\right)^{r} \frac{\Gamma(-\alpha-r)}{\Gamma(-\alpha)} \frac{\Gamma(L+r)}{\Gamma(L)},
$$

provided $\alpha<-r$, and infinite otherwise. To simplify calculation and with the intention of obtaining comparable results, in most experiments we deal with a restricted case which assumes $E(Z)=1$, in this case Eq. (2) implies that $\gamma^{*}=-\alpha-1$.

We are interested in simulating the noisiest case which occurs when $L=1$; it is called single-look and expression (1) becomes

$$
f_{Z}(z)=\frac{-\alpha}{\gamma}\left(\frac{z}{\gamma}+1\right)^{\alpha-1}
$$

In this case the cumulative distribution function is given by

$$
F_{Z}(z)=1-\left(\frac{z}{\gamma}+1\right)^{\alpha}
$$

In order to estimate the parameters $\alpha$ and $\gamma$, the maximum likelihood method is used. Given the sample $\boldsymbol{z}=\left(z_{1}, \ldots, z_{n}\right)$ of independent and identically distributed random variables with common distribution $\mathcal{G}_{I}^{0}(\alpha, \gamma, 1)$ with $(\alpha, \gamma) \in \Theta=\mathbb{R}_{-} \times \mathbb{R}_{+}$, a maximum likelihood estimator of $(\alpha, \gamma)$ satisfies

$$
(\widehat{\alpha}, \widehat{\gamma})=\arg \max _{(\alpha, \gamma) \in \Theta} \mathcal{L}(\alpha, \gamma, 1, \boldsymbol{z})
$$

where $\mathcal{L}$ is the likelihood function. This leads to $\widehat{\alpha}$ and $\widehat{\gamma}$ such that

$$
\begin{array}{r}
n\left[\Psi^{0}(-\widehat{\alpha})-\Psi^{0}(1-\widehat{\alpha})\right]+\sum_{i=1}^{n} \ln \frac{\widehat{\gamma}+z_{i}^{2}}{\widehat{\gamma}}=0 \\
\frac{n \widehat{\alpha}}{\widehat{\gamma}}+(1-\widehat{\alpha}) \sum_{i=1}^{n}\left(\widehat{\gamma}+z_{i}^{2}\right)^{-1}=0
\end{array}
$$

where $\Psi^{0}(t)=d \ln \Gamma(t) / d t$ is the digamma function.

\section{ENTROPIES FOR THE $\mathcal{G}_{I}^{0}$ DISTRIBUTION}

Let $f$ be a probability density function with support $\Omega$, its $(h, \phi)$ entropy $H_{\phi}^{h}(f, \theta)$ is given by:

$$
H_{\phi}^{h}(f, \theta)=h\left[\int_{\Omega} \phi(f(x, \theta)) d x\right],
$$

being $\phi:[0,+\infty) \rightarrow \mathbb{R}$ concave and $h: \mathbb{R} \rightarrow \mathbb{R}$ an increasing function or $\phi:[0,+\infty) \rightarrow \mathbb{R}$ convex and $h: \mathbb{R} \rightarrow \mathbb{R}$ a decreasing function (Salicrú et al., 1994).

In this work we use Shannon and Rényi entropies, with

- Shannon Entropy: $h(x)=x$ and $\phi(x)=-x \ln x$.

- Rényi Entropy: $h(x)=(1-\beta)^{-1} \ln x$ and $\phi(x)=x^{\beta}$, $\beta \in(0,1)$.

We calculate the entropies for the $\mathcal{G}_{I}^{0}(\alpha, \gamma, 1)$ distribution. The Shannon entropy for a probability density function $f$ with parameters $\theta=\left(\theta_{1}, \ldots, \theta_{n}\right)$ is given by:

$$
H_{S}(f, \theta)=-\int_{-\infty}^{+\infty} f(z, \theta) \ln f(z, \theta) d z .
$$

Then, for the $\mathcal{G}_{I}^{0}$ distribution, considering $\theta=(\alpha, \gamma)$ and $L=$ 1:

$$
\begin{aligned}
H_{S}\left(f_{\mathcal{G}_{I}^{0}}, \alpha, \gamma\right) & =-\int_{0}^{+\infty}-\frac{\alpha}{\gamma}\left(1+\frac{z}{\gamma}\right)^{\alpha-1} \\
& * \ln \left(-\frac{\alpha}{\gamma}\left(1+\frac{z}{\gamma}\right)^{\alpha-1}\right) d z \\
& =\frac{\alpha-1}{\alpha}-\ln \left(-\frac{\alpha}{\gamma}\right) .
\end{aligned}
$$

The Rényi entropy with $\beta$ parameter for any probability density function $f$ is given by:

$$
H_{R}^{\beta}(f, \theta)=\frac{1}{1-\beta} \ln \int_{-\infty}^{+\infty}[f(z)]^{\beta} d z, \beta \in(0,1) .
$$


And for the Single Look $\mathcal{G}_{I}^{0}$ distribution is given by

$$
\begin{aligned}
H_{R}^{\beta}\left(f_{\mathcal{G}_{I}^{0}}, \alpha, \gamma\right) & =\frac{\beta}{1-\beta} \ln \left(-\frac{\alpha}{\gamma}\right)+ \\
& \frac{1}{1-\beta} \ln \left(-\frac{\gamma}{\beta(\alpha-1)+1}\right)
\end{aligned}
$$

\subsection{Asymptotic Entropy Distribution}

Following the result from (Salicrú et al., 1994), we know that given $\widehat{\theta}=\left(\widehat{\theta}_{1}, \widehat{\theta}_{2}, \ldots, \widehat{\theta}_{p}\right)$ the maximum likelihood estimator of the parameters of a distribution $f(Z, \theta)$, based on a random sample of size $N$, then:

$$
\sqrt{N}\left(H_{h}^{\phi}(f, \widehat{\theta})-H_{h}^{\phi}(f, \theta)\right) \underset{N \rightarrow \infty}{\stackrel{D}{\longrightarrow}} \mathcal{N}\left(0, \sigma_{H}^{2}\right),
$$

being $\sigma_{H}^{2}(\theta)=\delta^{T} K(\theta)^{-1} \delta$, where $K(\theta)$ is the Fisher Information matrix of $f(Z, \theta)$ and $\delta$ is given by:

$$
\delta=\left[\delta_{1}, \delta_{2}, \ldots, \delta_{p}\right] \quad \text { with } \quad \delta_{i}=\frac{\partial H_{h}^{\phi}(\theta)}{\partial \theta_{i}}
$$

and

$$
K(\theta)=E\left(-\frac{\partial^{2} \ln f(z, \theta)}{\partial \theta^{2}}\right)
$$

From Eq. (3),

$$
\ln f(z, \alpha, \gamma)=\ln (-\alpha)-\ln \gamma+(\alpha-1) \ln \left(1+\frac{z}{\gamma}\right) .
$$

The second order derivatives are given by (Naranjo-Torres et al., 2017):

$$
\begin{aligned}
& \frac{\partial^{2} \ln f}{\partial \alpha^{2}}=-\frac{1}{\alpha^{2}} \\
& \frac{\partial^{2} \ln f}{\partial \alpha \partial \gamma}=-\frac{z}{\gamma^{2}+z \gamma} \\
& \frac{\partial^{2} \ln f}{\partial \gamma^{2}}=\frac{1}{\gamma^{2}}+\frac{\alpha-1}{\gamma^{2}}\left[-\frac{z^{2}}{(\gamma+z)^{2}}+\frac{2 z}{z+\gamma}\right]
\end{aligned}
$$

Then, the Fisher information matrix is given by:

$$
K(\theta)=\left(\begin{array}{cc}
\frac{1}{\alpha^{2}} & \frac{1}{\gamma(1-\alpha)} \\
\frac{1}{\gamma(1-\alpha)} & \frac{\alpha}{\gamma^{2}(\alpha-2)}
\end{array}\right)
$$

From (9), for the $\mathcal{G}_{I}^{0}(\alpha, \gamma, 1)$ distribution and the Shannon entropy, we have

$$
\delta_{S}^{T}=\left[\delta_{1}, \delta_{2}\right]=\left[\frac{\partial H_{S}(\alpha, \gamma)}{\partial \alpha}, \frac{\partial H_{S}(\alpha, \gamma)}{\partial \gamma}\right]
$$

then

$$
\delta_{S}^{T}=\left[\frac{1}{\alpha^{2}}-\frac{1}{\alpha}, \frac{1}{\gamma}\right]
$$

For the Rényi entropy:

$$
\begin{aligned}
\delta_{R}^{T} & =\left[\frac{\partial H_{R}(\alpha, \gamma)}{\partial \alpha}, \frac{\partial H_{R}(\alpha, \gamma)}{\partial \gamma}\right] \\
& =\left[\frac{r}{1-r}\left(\frac{1}{\alpha}-\frac{1}{r(\alpha-1)+1}\right), \frac{1}{\gamma}\right] .
\end{aligned}
$$

Then, the asymptotic variance of the Shannon entropy is given by:

$$
\sigma_{H_{S}}^{2}=\left(\frac{1}{\alpha^{2}}-\frac{1}{\alpha} ; \frac{1}{\gamma}\right)\left(\begin{array}{cc}
\frac{1}{\alpha^{2}} & \frac{\alpha}{\gamma^{2}(\alpha-1)} \\
\frac{\alpha}{\gamma^{2}(\alpha-1)} & \frac{\alpha}{\gamma^{2}(\alpha-2)}
\end{array}\right)^{-1}\left(\begin{array}{c}
\frac{1}{\alpha^{2}}-\frac{1}{\alpha} \\
\frac{1}{\gamma}
\end{array}\right)
$$

The Inverse of the Fisher Information Matrix expression for the $\mathcal{G}_{I}^{0}$ distribution is:

$$
[K(\theta)]^{-1}=\left(\begin{array}{cc}
(\alpha-1)^{2} \alpha^{2} & \alpha \gamma(\alpha-1)(\alpha-2) \\
\alpha \gamma(\alpha-1)(\alpha-2) & \gamma^{2}(\alpha-1)^{2}(\alpha-2) / \alpha
\end{array}\right)
$$

Then de asymptotic variance of Shannon Entropy expression is:

$\sigma_{H_{S}}^{2}=\frac{(\alpha-1)^{2}\left[\left(-2+4 \alpha^{3}-\gamma^{2}-\alpha^{2}\left(10+\gamma^{2}\right)+\alpha\left(5+2 \gamma^{2}\right)\right)\right]}{\alpha^{2}\left[\left(\alpha\left(5+2 \gamma^{2}\right)-2-\gamma^{2}-\alpha^{2}\left(2+\gamma^{2}\right)\right]\right.}$

and for the Rényi entropy:

$$
\begin{aligned}
\sigma_{H_{R}^{\beta}}^{2}= & \frac{(\alpha-1)^{2}\left(-2+4 \beta+4 \alpha^{3} \beta^{2}-\beta^{2}\left(4+\gamma^{2}\right)\right.}{(1+(\alpha-1) \beta)^{2}\left(\alpha\left(5+2 \gamma^{2}\right)-\alpha^{2}\left(2+\gamma^{2}\right)-\gamma^{2}-2\right)} \\
& +\frac{\alpha\left(1-10 \beta+2 \beta^{2}\left(7+\gamma^{2}-\alpha^{2} \beta\left(-4+\beta\left(14+\gamma^{2}\right)\right)\right)\right)}{(1+(\alpha-1) \beta)^{2}\left(\alpha\left(5+2 \gamma^{2}\right)-\alpha^{2}\left(2+\gamma^{2}\right)-\gamma^{2}-2\right)}
\end{aligned}
$$

being $\delta=\frac{\beta(\alpha-1)}{1-\beta}$ and $\lambda=1-\frac{\alpha}{\beta(\alpha-1)+1}$.

\subsection{Hypothesis Testing with $h-\phi$ entropies}

Let $X=\left\{x_{1}, \ldots, x_{N_{1}}\right\}$ and $Y=\left\{y_{1}, \ldots, y_{N_{2}}\right\}$ be two random samples from the $\mathcal{G}_{I}^{0}$ distribution and parameters $\left(\alpha_{1}, \gamma_{1}\right)$ and $\left(\alpha_{2}, \gamma_{2}\right)$, respectively. In order to analyze if $X$ and $Y$ come from the same distribution, it can be utilized the following hypothesis test:

$$
\begin{aligned}
& H_{0}: H_{\mathcal{M}}\left(\widehat{\theta}_{1}\right)=H_{\mathcal{M}}\left(\widehat{\theta}_{2}\right)=v \\
& H_{1}: H_{\mathcal{M}}\left(\widehat{\theta}_{1}\right) \neq H_{\mathcal{M}}\left(\widehat{\theta}_{2}\right),
\end{aligned}
$$

where $\mathcal{M} \in\{S, R\}, S$ and $R$ indicate Shannon and Rényi entropies, respectively.

Let $\widehat{\theta}_{i}$ be the maximum likelihood estimator of $\theta_{i}$ using a sample of size $N_{i}$. Following (Salicrú et al., 1994), for $r \geq 2$

$$
\sum_{i=1}^{r} \frac{N_{i}\left(H_{\mathcal{M}}\left(\widehat{\theta}_{i}\right)-\bar{v}\right)^{2}}{\sigma_{\mathcal{M}}^{2}\left(\widehat{\theta}_{i}\right)} \underset{N \rightarrow \infty}{\stackrel{D}{\longrightarrow}} \chi_{r-1}^{2}
$$

being

$$
\bar{v}=\left[\sum_{i=1}^{r} \frac{N_{i}}{\sigma_{\mathcal{M}}^{2}\left(\widehat{\theta}_{i}\right)}\right]^{-1} \sum_{i=1}^{r} \frac{N_{i} H_{\mathcal{M}}\left(\widehat{\theta}_{i}\right)}{\sigma_{\mathcal{M}}^{2}\left(\widehat{\theta}_{i}\right)}
$$

In our case $r=2, \theta=(\alpha, \gamma)$ and $N_{1}=N_{2}=N$, the test 
statistic is:

$$
S_{\mathcal{M}}\left(\theta_{1}, \theta_{2}\right)=N \frac{\left(H_{\mathcal{M}}\left(\widehat{\theta}_{1}\right)-H_{\mathcal{M}}\left(\widehat{\theta}_{2}\right)\right)^{2}}{\sigma_{\mathcal{M}}^{2}\left(\widehat{\theta}_{1}\right)+\sigma_{\mathcal{M}}^{2}\left(\widehat{\theta}_{2}\right)} .
$$

For large samples, $S_{\mathcal{M}}\left(\theta_{1}, \theta_{2}\right)=s$, the null hypothesis can be rejected with significance level $\eta$ if:

$$
P\left(\chi_{r-1}^{2}>s\right) \leq \eta,
$$

where $P\left(\chi_{r-1}^{2}>s\right)$ is the $p$-value for the statistic $S_{\mathcal{M}}, \mathcal{M} \in$ $\{S, R\}$. It can be observed that if the null hypothesis is rejected, it means that the samples are from different distributions with a significance level $\eta$.

\section{PROPOSAL ALGORITHM FOR SPECKLE REDUCTION}

In this section we explain the method for despeckling an image by means of testing if two samples $X \sim \mathcal{G}_{I}^{0}\left(\alpha_{1}, \gamma_{1}, 1\right)$ and $Y \sim \mathcal{G}_{I}^{0}\left(\alpha_{2}, \gamma_{2}, 1\right)$ have the same entropy and then they have been drawn from the same distribution, then we pose the null hypothesis $H_{0}: H_{\mathcal{M}}\left(\alpha_{1}, \gamma_{1}\right)=H_{\mathcal{M}}\left(\alpha_{2}, \gamma_{2}\right)$ and we want to know the probability of rejecting it. The test is based on the asymptotic distribution of $S_{\mathcal{M}}, \mathcal{M} \in\{S, R\}$.

The filter is built in the following way, we consider two sliding windows, $W_{i}, i=1, \ldots, n$ of size $t \times t$ (white) and $W_{j}^{i}, j=$ $1, \ldots, t^{2}$ of size $k \times k$ (light blue), where $k<<t$, as shown in Figure 1. For each $i \in\{1, \ldots, n\}$, a small window $W_{c}^{i}$ centered in the the central pixel of the window $W_{i}$ (violet), is considered. The pixels sample from $W_{c}^{i}$ is compared with the pixels samples of small sliding windows inside $W_{i}$.

The computed $p$-values of the statistics $S_{\mathcal{M}}, \mathcal{M} \in\{S, R\}$ are used as weights of a smoothing mask. Algorithm 1 shows the steps of the method.

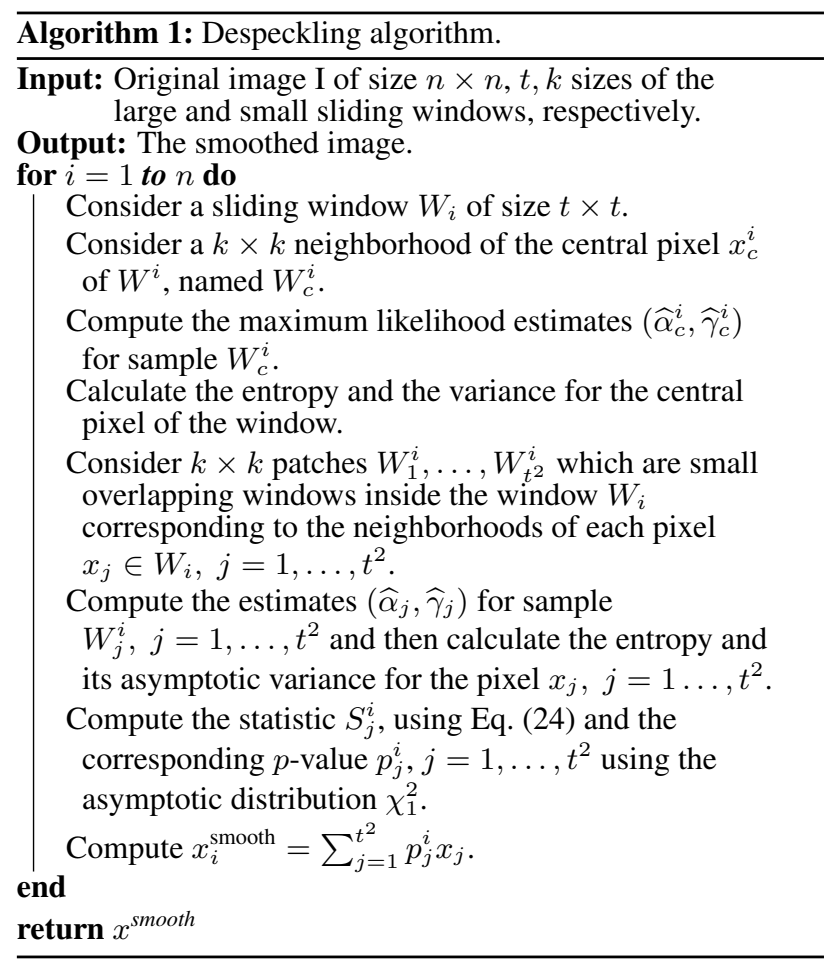

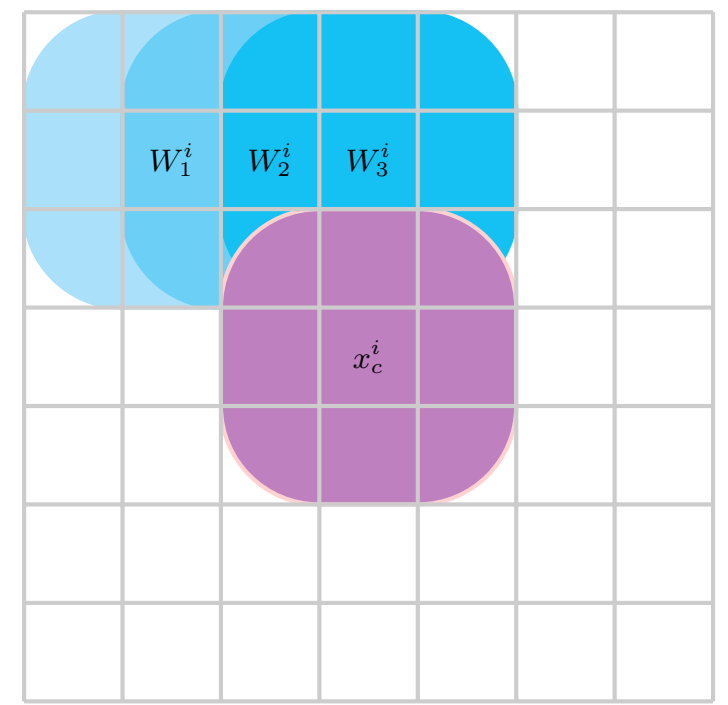

Figure 1. The large sliding window and small patches inside the window.

\section{RESULTS}

In order to assess the smoothing quality, we follow the proposal in (Gómez Déniz et al., 2017) and we compute the equivalent number of looks, and the average over the whole image. A good smoothing method should increase the equivalent number of looks, and retain the average of whole original image in the resulting image.

Figure 2 shows the result of applying the method to a simulated image generated under the $\mathcal{G}_{I}^{0}$ distribution. For this construction we apply the recommendations of (Chan et al., 2018). Figure 2(a) shows the original data, the two halves of the image were generated with $\mathcal{G}_{I}^{0}(-4,3,1)$ and $\mathcal{G}_{I}^{0}(-1.5,0.5,1)$ distributions, respectively. Figure 2(b) shows the filtered image with patches of size $5 \times 5$, a sliding window of size $25 \times 25$ and the statistic is computed with the Rényi entropy.

For cases where reference ground truth image is not available, the estimations of image mean preservation and variance reduction, estimated in a user-selected homogeneous area, are necessary for assessing the despeckling quality of the methodology.

In the smoothed image the ENL should be as high as possible, because a high ENL value reveals a greater speckle rejection and therefore an improved ability to distinguish different texture levels.

Given an image I and considering an homogeneous region $I_{h}$, with intensity average value $\bar{z}$ and a standard deviation $s$, its ENL is estimated:

$$
E N L=\frac{\mu^{2}}{\sigma^{2}}
$$

Table 1 shows the estimates of the equivalent number of looks (ENL) and average for the original and the smoothed images. It can be observed the increment in the ENL value while the average is almost the same.

Figure 3 shows the result of applying the method to a synthetic image generated with the $\mathcal{G}_{I}^{0}$ distribution. Figure 3(a) shows the original data, the four regions of the image are generated with $\mathcal{G}_{I}^{0}(-5,10,1), \mathcal{G}_{I}^{0}(-5,1,1), \mathcal{G}_{I}^{0}(-1.5,10,1)$ and 


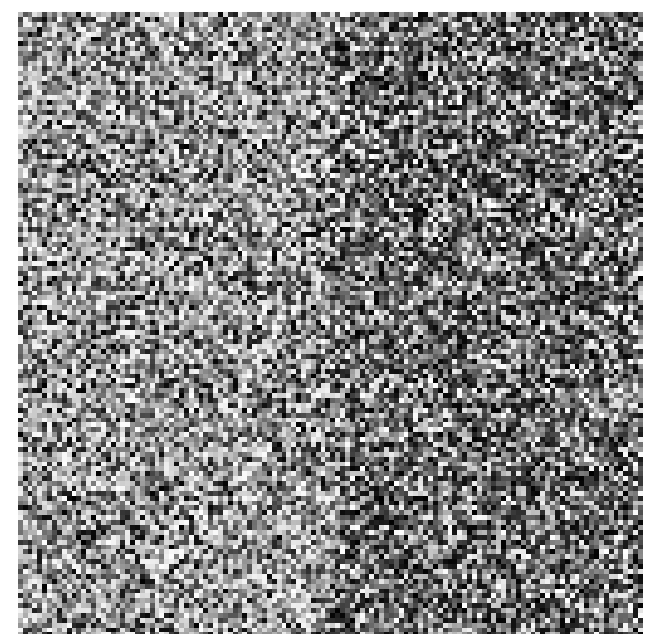

(a) Original image.

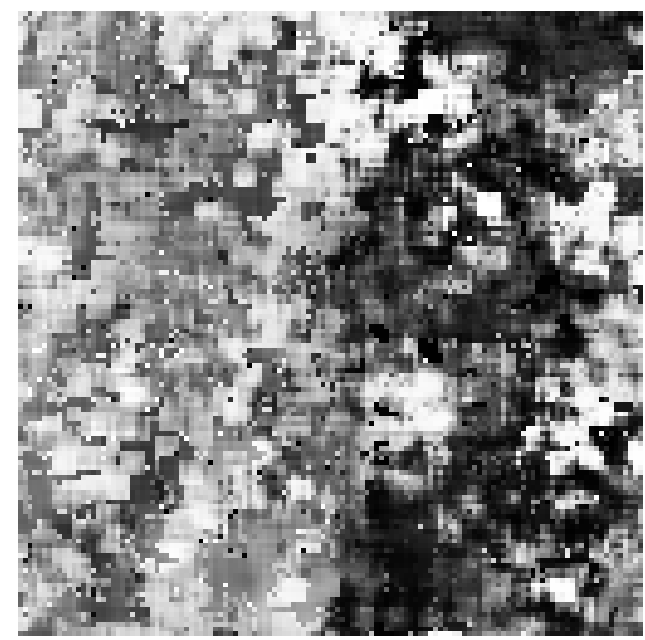

(b) Despeckled image.

Figure 2. Result of applying the despeckling method using Rényi entropy.

$\mathcal{G}_{I}^{0}(-1.5,1,1)$ distributions. Figure 3(b) shows the filtered image with a small windows of size $7 \times 7$, a large window of size $49 \times 49$ and the statistic is computed with the Shannon entropy.

Table 2 shows the estimates of the equivalent number of looks and average for the original and the smoothed images. It can be observed the increment in the ENL value while the average keeps the same.

\section{CONCLUSIONS AND FUTURE WORK}

We have proposed a new nonlocal means filter for single-look speckled data based on the asymptotic distribution of the Shannon and Rényi entropies for the $\mathcal{G}_{I}^{0}$ distribution.

The measure of similarity between the central window and the patches is based on a test statistic for checking if the two samples come from the same distribution.

Table 1. Estimates of the equivalent number of looks for both images from Figure 2.

\begin{tabular}{crr}
\hline Figure & ENL & Average \\
\hline 2(a) & 0.85 & 1.04 \\
2(b) & 58.36 & 0.96 \\
\hline
\end{tabular}

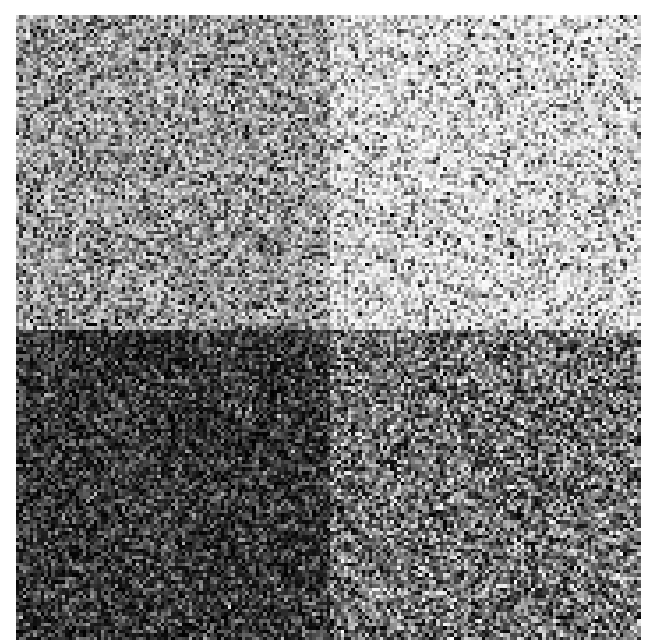

(a) Original image.

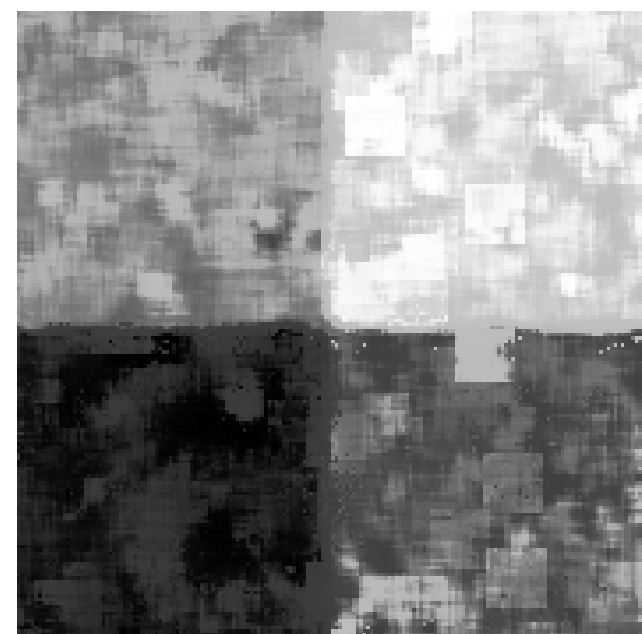

(b) Despeckled image.

Figure 3. Result of applying the despeckling method using Shannon entropy.

The results are encouraging, as the filtered image has better signal-to-noise ratio, it preserves the mean, and the edges are not severely blurred.

In future works, we want to asses with several criteria the performance of this filter in case of contaminated data and we will consider another measures as Kullback-Leibler distance and test its performance for a similar construction of a filter on actual SAR data.

\section{REFERENCES}

Buemi, M. E., Frery, A. C., Ramos, H. S., 2014. Speckle reduction with adaptive stack filters. Pattern Recognition Letters, 36, 281-287. https://doi.org/10.1016/j.patrec.2013.06.005.

Chan, D., Rey, A., Gambini, J., Frery, A. C., 2018. Sampling

Table 2. Estimates of the equivalent number of looks for both images from Figure 3.

\begin{tabular}{lrr}
\hline Figure & ENL & Average \\
\hline 2(a) & 0.88 & 0.25 \\
2(b) & 37.27 & 0.25 \\
\hline
\end{tabular}


from the $\mathcal{G}_{I}^{0}$ distribution. Monte Carlo Methods and Applications.

Chan, D., Rey, A., Gambini, J., Frery, A. C., 2019. Low-Cost Robust Estimation for the Single-Look $\mathcal{G}_{I}^{0}$ Model Using the Pareto Distribution. IEEE Geoscience and Remote Sensing Letters.

Dellamonica, D., Silva, P. J., Humes, C., Hirata, N. S., Barrera, J., 2007. An exact algorithm for optimal mae stack filter design. IEEE transactions on image processing, 16(2), 453-462.

Ferraioli, G., Pascazio, V., Schirinzi, G., 2019. Ratio-Based Nonlocal Anisotropic Despeckling Approach for SAR Images. IEEE Transactions on Geoscience and Remote Sensing, 57(10), 7785-7798.

Frery, A., Müller, H., Yanasse, C., Sant'Anna, S., 1997. A model for extremely heterogeneous clutter. IEEE Transactions on Geoscience and Remote Sensing, 35(3), 648-659.

Gambini, J., Cassetti, J., Lucini, M., Frery, A., 2015. Parameter Estimation in SAR Imagery using Stochastic Distances and Asymmetric Kernels. IEEE Journal of Selected Topics in Applied Earth Observations and Remote Sensing, 8(1), 365375.

Gao, G., 2010. Statistical modeling of SAR images: A survey. Sensors, 10(1), 775-795.

Gómez Déniz, L., Ospina, R., Frery, A. C., 2017. Unassisted Quantitative Evaluation of Despeckling Filters. Remote Sensing, 9(4), 1-23. https://doi.org/10.3390/rs9040389.

Kuan, D., Sawchuk, A., Strand, T., Chavel, P., 1987. Adaptive restoration of images with speckle. IEEE Transactions on Acoustics, Speech, and Signal Processing, 35(3), 373-383.

Kullback, S., Leibler, R. A., 1951. On Information and Sufficiency. Ann. Math. Statist., 22(1), 79-86. https://doi.org/10.1214/aoms/1177729694.

Lebrun, M., Buades, A., Morel, J., 2013. A Nonlocal Bayesian Image Denoising Algorithm. SIAM J. Imaging Sciences, 6(3), 1665-1688. https://doi.org/10.1137/120874989.

Liese, F., Vajda, I., 2006. On Divergences and Informations in Statistics and Information Theory. IEEE Transactions on Information Theory, 52, 4394-4412.

Lin, J.-H., Kim, Y.-T., 1994. Fast algorithms for training stack filters. IEEE Transactions on Signal Processing, 42(4), 772781.

Lin, J.-H., Sellke, T. M., Coyle, E. J., 1990. Adaptive stack filtering under the mean absolute error criterion. IEEE transactions on acoustics, speech, and signal processing, 38(6), 938954.

Lopes, A., Nezry, E., Touzi, R., Laur, H., 1990. Maximum a posteriori speckle filtering and first order texture models in sar images. Geoscience and Remote Sensing Symposium, 1990. IGARSS'90.'Remote Sensing Science for the Nineties'., 10th Annual International, Ieee, 2409-2412.

Mejail, M., Berlles, J. J., Frery, A. C., Bustos, O., 1998. Parametric Roughness Estimation in Amplitude: SAR Images Under the Multiplicative Model. Universidad Nacional de Cordoba. Instituto de Matematica, Astronomia y Fisica.
Naranjo-Torres, J., Gambini, J., Frery, A. C., 2017. The Geodesic Distance between $\mathcal{G}_{I}^{0}$ Models and its Application to Region Discrimination. IEEE Journal of Selected Topics in Applied Earth Observations and Remote Sensing, 10(3), 987-997.

Nascimento, A. D. C., Frery, A. C., Cintra, R. J., 2018. Detecting Changes in Fully Polarimetric SAR Imagery with Statistical Information Theory. CoRR, abs/1801.08901. http://arxiv.org/abs/1801.08901.

Oliver, C., Quegan, S., 2004. Understanding synthetic aperture radar images. SciTech Publishing.

Rényi, A., 1961. On measures of entropy and information. Proceedings of the Fourth Berkeley Symposium on Mathematical Statistics and Probability, Volume 1: Contributions to the Theory of Statistics, University of California Press, Berkeley, Calif., 547-561.

Rojo, J., 2013. Heavy-tailed densities. Wiley Interdisciplinary Reviews: Computational Statistics, 5(1), 30-40.

Salicrú, M., Morales, D., Menéndez, M. L., Pardo, L., 1994. On the Applications of Divergence Type Measures in Testing Statistical Hypotheses. Journal of Multivariate Analysis, 51(2), 372-391.

Sant'Anna, S. J. S., 1995. Avaliação do desempenho de filtros redutores de speckle em imagens de radar de abertura sintética. Master's thesis, Instituto Nacional de Pesquisas Espaciais.

Shannon, C. E., 1948. A Mathematical Theory of Communication. The Bell System Technical Journal, 27, 379-423.

Torres, L., Sant'Anna, S. J. S., Freitas, C. C., Frery, A. C., 2014. Speckle Reduction in Polarimetric SAR Imagery with Stochastic Distances and Nonlocal Means. Pattern Recognition, 47, 141-157.

Wang, Z., Bovik, A. C., Lu, L., 2002. Why is image quality assessment so difficult? ICASSP, 4, 3313-3316. 